\title{
Emissivity of Hydrogen Atoms at High Temperatures*
}

\author{
Henry Aroeste and William C. Benton \\ Daniel and Florence Guggenheim Jet Propulsion Center, California Institute of Technology, Pasadena, California
}

(Received August 12, 1955)

\begin{abstract}
A method is described for making estimates of the total emissivity of hydrogen in the temperature and pressure ranges where hydrogen atoms predominate under equilibrium conditions. For a typical geometrical depth of $50 \mathrm{~cm}$, and temperatures of the order of $12500^{\circ} \mathrm{K}$ and higher, with pressures of the order of 100 atmos and higher, the emissivity approaches unity $(\epsilon>0.95)$, while for temperatures of the order of $9500^{\circ} \mathrm{K}$ and lower, with pressures of the order of 10 atmos and lower, the emissivity approaches zero $(\epsilon \widetilde{<} 0.05)$. The variations of the emissivity between these approximate limits are shown graphically as functions of temperature and pressure with the geometrical depth set at $50 \mathrm{~cm}$. The variation of the emissivity with geometrical depth is also shown graphically at $12600^{\circ} \mathrm{K}$ and 20 atmos.
\end{abstract}

\section{INTRODUCTION}

I $T$ is of some interest to obtain the emissivity of hydrogen atoms as a function of temperature and pressure in order to assess the importance of radiant

* Supported by the Office of Naval Research under Contract Nonr-220(03), NR 015401 . Reproduction in whole or in part is permitted for any purpose of the United States Government. Previous publications of this series have been concerned with heat transfer for high-temperature systems utilizing hydrogen. If the temperatures are sufficiently high, the hydrogen will exist almost completely in the atomic

emissivity calculations on diatomic and polyatomic molecules [see S. S. Penner, J. Appl. Phys. 25, 660 (1954) and earlier papers].

† This article uses, in part, the results of a thesis submitted by William C. Benton, Major, U. S. Marine Corps, in partial fulfillment of requirements for the degree of Aeronautical Engineer, California Institute of Technology, June, 1955. 


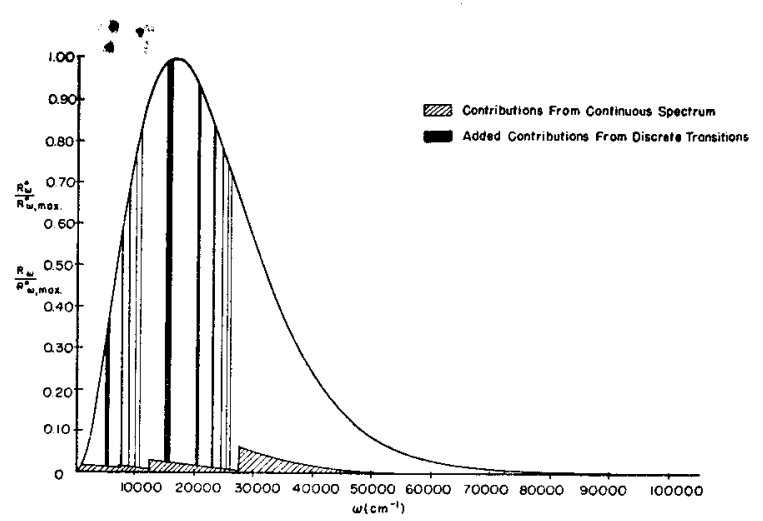

FIG. 1. Plot for determining emissivity at $8400^{\circ} \mathrm{K}$ and 40 atmos $(l=50 \mathrm{~cm})$.

state, and hydrogen atoms will make the major contribution to the total emissivity. Furthermore, the treatment for hydrogen atoms provides a simple example of emissivity calculations for atomic systems.

The emissivity $\epsilon$ of a body is the ratio of the emitted radiant energy to the radiant energy emitted by a blackbody. The contributions to the emissivity of hydrogen atoms come from two sources: the continuous spectrum and the discrete transitions.

The blackbody curves may be obtained by plotting

$$
R_{\omega}{ }^{0}=\frac{2 \pi k C^{2} \omega^{3}}{\exp (k c \omega / k T)-1}
$$

against $\omega$ for various temperatures. Here $R_{\omega}{ }^{0} d \omega$ (erg/ $\mathrm{cm}^{2}-\mathrm{sec}$ ) is the radiant energy emitted by a blackbody, per unit area, per unit time, in the wave number interval $d \omega$, into a solid angle of $2 \pi$ steradians, $T\left({ }^{\circ} \mathrm{K}\right)$ is the absolute temperature, $C(\mathrm{~cm} / \mathrm{sec})$ is the velocity of light, $k$ (erg-sec) is Planck's constant, and $k\left(\mathrm{erg} /{ }^{\circ} \mathrm{K}\right.$ ) is the Boltzmann constant. If one considers the system of heated hydrogen atoms to be isothermal and uniform, then $R_{\omega}=R_{\omega}^{0}\left[1-\exp \left(P_{\omega} X\right)\right]$. This function can be plotted as a function of $\omega$ on the same graphs as $R^{0}{ }_{\omega}$. Here $R_{\omega} d \omega\left(\mathrm{erg} / \mathrm{cm}^{2}-\mathrm{sec}\right)$ is the radiant energy emitted by the hydrogen atoms, per unit area, per unit time, in the wave number interval $d \omega$, into a solid angle of $2 \pi$

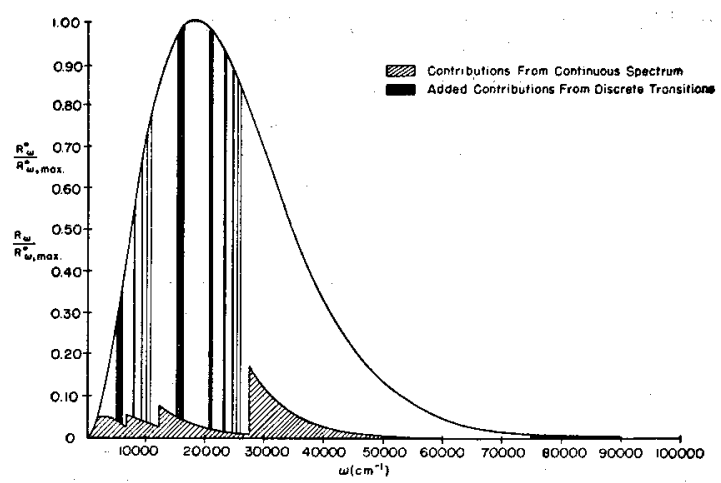

FIG. 2. Plot for determining emissivity at $9200^{\circ} \mathrm{K}$ and 40 atmos $(l=50 \mathrm{~cm})$. steradians, $P_{\omega}\left(\mathrm{cm}^{-1}\right.$-atmos $\left.{ }^{-1}\right)$ is the spectral absorption coefficient, and $X$ (cm-atmos) is the optical density. The spectral absorption coefficient $P_{\omega}$ is set equal to $P_{\omega}{ }^{d}+P_{\omega}{ }^{c}$, where $P_{\omega}{ }^{d}$ arises from discrete transitions and $P_{\omega}{ }^{c}$ from continuous spectrum contributions. To obtain the emissivity one takes the ratio of the actual area under the $R_{\omega}$ vs $\omega$ curve to the blackbody area, $\sigma T^{4}$, where $\sigma=5.6699 \times 10^{-5} \mathrm{erg} / \mathrm{cm}^{2}{ }^{\circ} \mathrm{K}^{4}$-sec. Examples of plots of $R_{\omega}$ and $R_{\omega}^{0}$ as functions of $\omega$ are reproduced in Figs. 1 to 5 for various temperatures at a representative pressure of 40 atmos. For convenience of presentation $R_{\omega}^{0} / R_{\omega, \max }^{0}$ have been used as ordinates, where $R_{\omega, \max }^{0}$ is the maximum value of $R^{0}{ }_{\omega}$. The considerations used for the calculation of $P_{\omega}$ and hence $R_{\omega}$ will now be described.

\section{EVALUATION OF THE SPECTRAL ABSORPTION COEFFICIENT $P_{\omega}$ AND OF OPTICAL DENSITY}

\section{A. Calculation of $\boldsymbol{P}_{\omega}$}

The mass absorption coefficient in $\mathrm{cm}^{2}$ per gram of neutral hydrogen atoms for the continuous spectrum, $X^{\prime}{ }_{p}$, has been given by Unsöld ${ }^{1}$ as

$$
X^{\prime}=\frac{64 \pi^{4} m q^{10}}{3 \sqrt{3} c k^{3} m_{H} k^{3}} \frac{e^{-u_{1}}}{T^{3} u^{3}}\left\{\sum_{u_{n}<u}^{u_{4}} \frac{e^{u_{n}}}{n^{3}}+\frac{e^{u_{5}}}{2 u_{1}}\right\}\left(1-e^{-u}\right) .
$$

Here $m$ is the mass of the electron $(g), q$ is the charge of the electron (esu), $m_{H}$ is the mass of the hydrogen atom $(g), u=h c \omega / k T, u_{n}=R h c / n^{2} k T, R$ is the Rydberg constant $\left(\mathrm{cm}^{-1}\right)$, and $n$ is the principal quantum number of the hydrogen atom. To convert $X^{\prime}{ }_{\nu}$ to $P_{\omega}{ }^{e}$, which has the units of $\mathrm{cm}^{-1}$-atmos ${ }^{-1}$, one must multiply by the atomic weight of hydrogen and divide by the absolute temperature and the molar gas constant, $R^{\prime}$, in $\mathrm{cm}^{3}$ atmos $/ \mathrm{mole}^{\circ}{ }^{\circ} \mathrm{K}$.

The contribution of the $K$ th spectral line whose center lies at $\omega$ can generally be determined by computing the maximum value of $R_{\omega}(K)=R_{\max }(K)$, which is usually the value of $R^{0}{ }_{\omega}(K)$ at the line center $\left[\equiv R_{\omega_{0}}^{0}(K)\right]$, and multiplying this quantity by the intensity width defined as the wave number range for which $R_{\omega}(K)$ $\geqslant \frac{1}{2} R_{\max }(K)$. Detailed calculations by the authors have shown that this procedure will give only an error of approximately one percent for the contribution of the line to the total emissivity. For collected methods of computing the spectral absorption coefficient of isolated lines see, for example, Penner and Kavanagh. ${ }^{2}$ The maximum absorption coefficient for purely Doppler broadened lines, $P^{\prime}$, is given by the equation

$$
P^{\prime}=\left(S / \omega_{0}\right)\left(m_{H} c^{2} / 2 \pi k T\right)^{\frac{2}{2}},
$$

where $S\left(\mathrm{~cm}^{-2}\right.$-atmos $\left.{ }^{-1}\right)$ is the integrated intensity of the spectral line under consideration. The integrated

IA. Unsöld, Physik der Sternatmospharen (Verlag Julius Springer, Berlin, 1938).

${ }^{2}$ S. S. Penner and R. W. Kavanagh, J. Opt. Soc. Am. 43, 385 (1953). See also reference 1. 
intensity $S$ may be written as

$$
S=S^{\prime}\left(N_{L} / N\right)\left[1-\exp \left(-h c \omega_{0} / k T\right)\right],
$$

where $N$ is the total number of atoms per $\mathrm{cm}^{3}$ per atmos, $N_{L}$ is the number of atoms in the lower state per $\mathrm{cm}^{3}$ per atmos. Here $S^{\prime}$ is given by

$$
S^{\prime}=\pi q^{2} N f / m c^{2},
$$

where $f$ is the "oscillator strength," which has been tabulated by Unsöld. ${ }^{1}$ At equilibrium

$$
N_{L} / N=\left(g_{L} / Q\right) \exp \left(-h c \omega_{L} / k T\right),
$$

where $g_{L}$ is the degeneracy of the lower state involved in the transition and $Q$ is the partition function. In this case $g_{L} / Q$ is taken as $n^{2}$ and $\omega_{L}=R\left(1-1 / n^{2}\right)$ where $n$ is the principal quantum number of the lower state in the transition. In the present calculation the partition function is, for practical purposes, equal to the contribution from the ground state. For the temperatures and pressures considered here it may be shown, using the

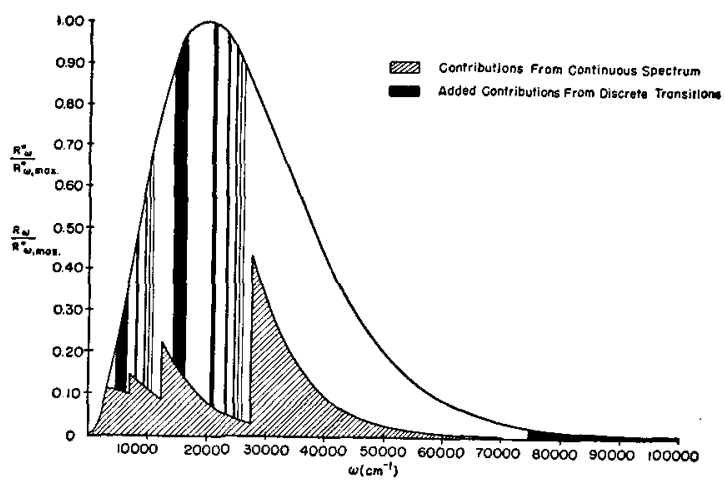

FIG. 3. Plot for determining emissivity at $10080^{\circ} \mathrm{K}$ and 40 atmos $(l=50 \mathrm{~cm})$

estimates of Urey, ${ }^{3}$ that this procedure does not introduce errors greater than about one percent. It is now possible to write Eq. (3) as

$$
\begin{aligned}
S=S^{\prime} n^{2} \exp \left[\left(1-1 / n^{2}\right)(-R h c / k T)\right] & \\
& \times\left[1-\exp \left(-h c \omega_{0} / k T\right)\right] .
\end{aligned}
$$

To estimate the intensity width, $2\left|\omega_{h}-\omega_{0}\right|$, at half the value of $R_{\max }(K)$, one uses the well-known curves of growth (see, for example, Unsöld ${ }^{1}$ or Penner and Kavanagh ${ }^{2}$. Here $\omega_{h}$ is a wave number such that $R_{\omega}(K)=\frac{1}{2} R_{\max }(K)$. The line shape parameter $a$ is given by

$$
a=b_{c}(\ln 2)^{\frac{1}{2}} / b_{D},
$$

where the natural half-width has been neglected compared to the collision half-width, $b_{c}$. The Doppler halfwidth, $b_{D}$, is given by

$$
b_{D}=\left(2 k T \ln 2 / m_{H} c^{2}\right)^{\frac{1}{2}} \omega_{0} .
$$

${ }^{8}$ H. C. Urey, Astrophys. J. 59, 1 (1924).

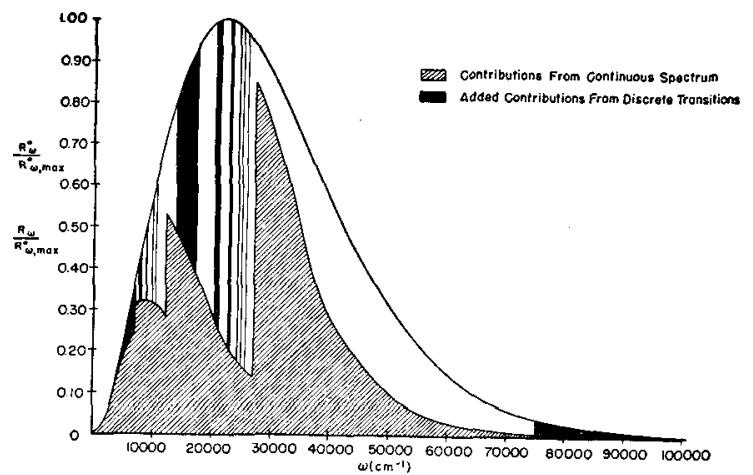

Fig. 4. Plot for determining emissivity at $11300^{\circ} \mathrm{K}$ and 40 atmos $(l=50 \mathrm{~cm})$

An estimate of the collision half-width is obtained from the formula of Weisskopf ${ }^{4}$ :

$$
b_{c}=q^{2} n f / 2 \pi m c^{2} \omega_{0},
$$

where $n$ is the number of molecules per $\mathrm{cm}^{3}$. Combining Eqs. (4) and (9), one obtains

$$
b_{c}=S^{\prime} P_{T} / 2 \pi^{2} \omega_{0}
$$

where $P_{T}$ is the pressure in atmos. Knowing $a$ and $P^{\prime} X$, it is possible to read from the curves of growth the value of the quantity $\zeta$,

where

$$
\zeta=A(\ln 2)^{\frac{1}{2}} / 2 R_{\omega}^{0} b_{D}
$$

$$
A=2\left|\omega_{h}-\omega_{0}\right| R_{\omega}^{0} .
$$

Combining Eqs. (11) and (12), one obtains

$$
\left|\omega_{h}-\omega_{0}\right|=\zeta b_{D} /(\ln 2)^{\frac{1}{2}} \text {. }
$$

If the Stark effect due to ionization is considered, $P_{\omega}{ }^{d}$ may be taken as a first rough estimate, as $P_{\omega}{ }^{S}+P_{\omega}{ }^{n}$, where $P_{\omega} S$ is the absorption coefficient due to ionization broadening alone, and $P_{\omega}{ }^{n}$ is the absorption coefficient due to broadening without considering ionization. A relation for the absorption coefficient due to ionization

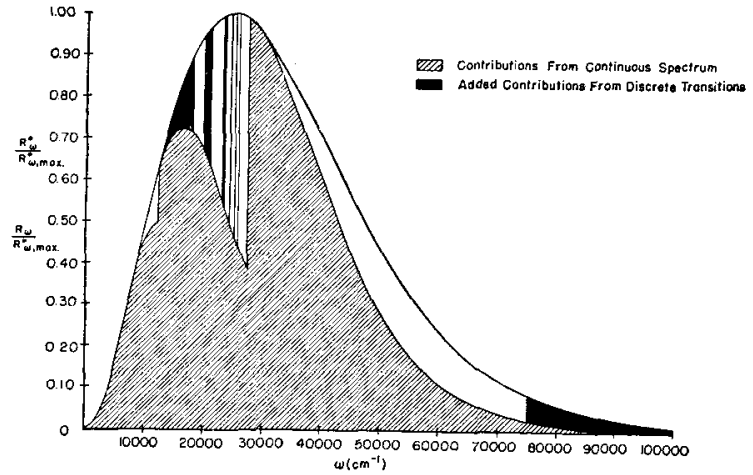

FIG. 5. Plot for determining emissivity at $12600^{\circ} \mathrm{K}$ and 40 atmos
$(l=50 \mathrm{~cm})$.

${ }^{4}$ H. Margenau and W. W. Watson, Revs. Modern Phys. 8, 41 (1936). 


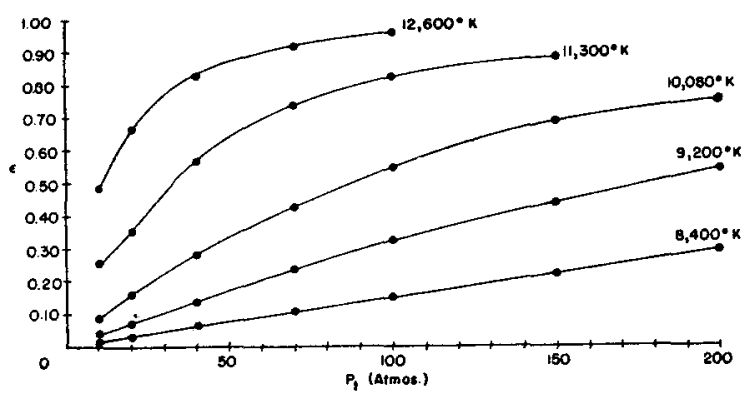

FIG. 6. Emissivity of hydrogen atoms at various temperatures as a function of total pressure $(l=50 \mathrm{~cm})$.

broadening is given by

$$
P_{\omega} S=\frac{\alpha(\Delta \lambda) n^{2} \exp \left(-h c \omega_{l} / k T\right) N_{0}}{R^{\prime} T},
$$

where $N_{0}$ is Avogadro's number, and $\alpha(\Delta \lambda)$ ( $\mathrm{cm}^{2} /$ atom) is the absorption coefficient per absorbing atom at a wavelength $\Delta \lambda(A)$ from $1 / \omega$. The relation for $\alpha(\Delta \lambda)$ is

$$
\alpha(\Delta \lambda)=4.21 C_{n} q^{3 / 2} N^{\prime} /(\Delta \lambda)^{5 / 2}{ }^{5}
$$

where $N^{\prime}$, the number of protons per $\mathrm{cm}^{3}$, may be determined from the Saha equation, and $C_{n}$ has been tabulated by Aller ${ }^{6}$ and Unsöld. ${ }^{1}$ Using Eqs. (14) and (15), a check of ionization broadening has been made at various temperatures and pressures using $H_{\alpha}$ as an example. In all cases it was found that $\left[1-\exp \left(-P_{\omega}{ }^{S} X\right)\right] \ll 1$ at the value of $\omega_{h}$ previously determined without considering Stark broadening, so that it seems justifiable to take $P_{\omega}{ }^{n}=P_{\omega}{ }^{d}$.

The method described above to determine contributions to the emissivity due to discrete transitions does not apply in the case of the Lyman lines because they are too intense, and the blackbody curve along the

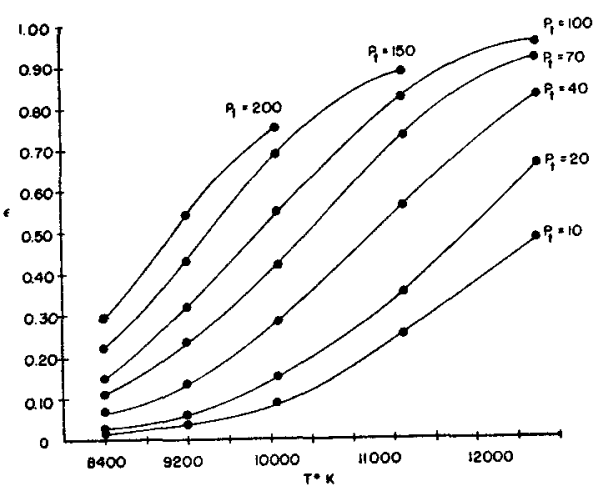

F1G. 7. Emissivity of hydrogen atoms at various total pressures as a function of temperature $(l=50 \mathrm{~cm})$.

'For a more complete discussion of the Stark effect due to ionization see reference 5 . Here broadening due to electrons is neglected The electron effect has been discussed recently by Kivel, Bloom, and Margenau, Phys. Rev. 98, 495 (1955).

6 L. H. Aller, The Atmospheres of the Sun and Stars (Ronald Press Company, New York, 1953), p. 311. See also reference 1. intensity width of the lines varies appreciably in this area. At the temperatures considered here it is only necessary to determine modifications of the above method for Lyman $\alpha$, since the succeeding Lyman lines give negligible contributions because of the small value of the blackbody radiancy $R_{\omega}{ }^{0}$ at large $\omega$. When $\left(\omega-\omega_{D}\right)$ is, for example, of such magnitude that it does not overlap an adjoining line, an expression for the discrete pressure broadening absorption coefficient, $P_{\omega}{ }^{n}$, has been given by Born. ${ }^{2}$ For large values of $\xi / a$, an asymptotic form of this relation is given by

$$
P_{\omega}{ }^{n} / P^{\prime}=a / \sqrt{ } \pi \xi^{2},
$$

where $\xi=\left[\left(\omega-\omega_{0}\right) / b_{D}\right](\ln 2)^{\frac{1}{2}}$. When $\left(\omega-\omega_{0}\right)$ is, for example, of such magnitude that it overlaps an adjoining line, Born's method for pressure broadening and the treatment described above for the Stark effect do not apply because one can no longer consider the contributions as resulting from "small" perturbations of welldefined energy states. The problem of the very broad Lyman $\alpha$ line has caused difficulty in related astrophysical applications, and an exact treatment is not feasible at present. Therefore, a reasonable cut-off point has been chosen beyond which the above treatment is not justifiable. The cut-off point to the left of the line is taken arbitrarily as half the distance between the Lyman $\alpha$ and Lyman $\beta$ line centers. The cut-off point to the right of the line is unimportant since the blackbody contribution is negligibly small. The application of Eqs. (14), (15), and (16) to the Lyman lines results effectively in adding a small contribution of blackbody radiation from the left cut-off point to $\omega=\infty$. It is felt that the neglect of the Lyman $\alpha$ contribution from $\omega=0$ to the left cut-off point does not give appreciable error, but this approximation may well deserve further investigation.

The contributions due to the higher series, Brackett, Pfund, etc., have not been considered in the present computations. These series will give appreciably contributions only at much lower temperatures than were used. Even if these contributions were blackbody from the left of the first Paschen line to $\omega=0$, the emissivity would in no case be increased by more than about $1 \%$.

\section{B. Determination of Optical Density $X$}

The optical density $X$ is defined as $p_{H} l$ where $p_{H}$ is the partial pressure of the hydrogen atoms (atmos) and $l$ is the geometric depth $(\mathrm{cm})$. The partial pressure is

TABLE I. Fraction of $\mathrm{H}_{2}$ dissociated $(X)$ and partial pressure

\begin{tabular}{|c|c|c|c|c|c|c|c|c|c|c|}
\hline \multirow[b]{2}{*}{$P_{T}$} & \multicolumn{2}{|c|}{$8400^{\circ} \mathrm{K}$} & \multicolumn{2}{|c|}{$9200^{\circ} \mathrm{K}$} & \multicolumn{2}{|c|}{$10080^{\circ} \mathrm{K}$} & \multicolumn{2}{|c|}{$11300^{\circ} \mathrm{K}$} & \multicolumn{2}{|c|}{$12600^{\circ} \mathrm{K}$} \\
\hline & $X$ & $p_{H}$ & $X$ & $p_{H}$ & $X$ & $p_{H}$ & $X$ & $p_{H}$ & $X$ & $p H$ \\
\hline $\begin{array}{r}50 \\
100 \\
150 \\
200\end{array}$ & $\begin{array}{l}0.976 \\
0.953 \\
0.932 \\
0.914\end{array}$ & $\begin{array}{r}49.5 \\
97.5 \\
145 \\
191\end{array}$ & $\begin{array}{l}0.974 \\
0.961 \\
0.950\end{array}$ & $\begin{array}{l}98.6 \\
147 \\
195\end{array}$ & $\begin{array}{l}0.984 \\
0.977 \\
0.968\end{array}$ & $\begin{array}{l}99.0 \\
148 \\
197\end{array}$ & $\begin{array}{l}0.986 \\
0.983\end{array}$ & $\begin{array}{l}149 \\
198\end{array}$ & 0.990 & 199 \\
\hline
\end{tabular}
in atmospheres of atomic hydrogen $\left(p_{H}\right)$ at various temperatures and total pressures. 
given as

$$
p_{H}=\frac{2 x}{1+x} P_{T}
$$

where $x$ is the degree of dissociation of hydrogen molecules and $P_{T}$ is the total pressure. The degree of dissociation $x$, may be determined from

$$
K_{p}=\left(4 x^{2} / 1-x^{2}\right) P_{T},
$$

where $K_{p}$ is the equilibrium constant for the dissociation of molecular hydrogen. Good estimates of $K_{p}$ can be obtained by extrapolating data given in the National Bureau of Standards Tables. ${ }^{7}$ Values of $x$ and $p_{H}$ are listed in Table I. From this table it is seen that hydrogen atoms may be expected to contribute significantly to the total emissivity. In a more exact treatment it would be necessary to consider the contributions of species other than hydrogen atoms which are present in relatively small amounts at the temperatures and pressures considered in the present investigations.

\section{RESULTS}

By use of the above procedures the total emissivity of hydrogen atoms in equilibrium with hydrogen molecules at various temperatures and pressures has been computed. For most of the computations a typical geometrical depth of $50 \mathrm{~cm}$ has been used, and Figs. 1 to 7 refer to that depth, as does the following discussion except where explicitly noted otherwise. In the ranges of temperatures and pressures chosen, the emissivity varies significantly with changes in the state variables. Typical plots used to determine the emissivity are shown in Figs. 1 to 5 , where the outer curve represents the blackbody curve, the cross-hatched area represents contributions from the continuous spectrum, and the solid area represents added contributions from the discrete transitions. The discontinuities in the continuous spectrum portions of Figs. 1 to 5 correspond to the onsets of the various continua; the Pfund continuum begins to contribute at $\omega=4380$, the Brackett continuum at $\omega=6850$, the Paschen continuum at $\omega=12190$ and the Balmer continuum at $\omega=27400$. It is not

TABLE II. Emissivity of atomic hydrogen as a function of temperature and total pressure $(l=50 \mathrm{~cm})$.

\begin{tabular}{cccccc}
\hline \hline $\operatorname{Pr}$ (atmos) & $\begin{array}{c}8400^{\circ} \mathrm{K} \\
\epsilon\end{array}$ & $\begin{array}{c}9200^{\circ} \mathrm{K} \\
\epsilon\end{array}$ & $\begin{array}{c}10080^{\circ} \mathrm{K} \\
\epsilon\end{array}$ & $\begin{array}{c}11 \\
\epsilon\end{array}$ & $\begin{array}{c}300^{\circ} \mathrm{K} \\
\epsilon 2600^{\circ} \mathrm{K} \\
\epsilon\end{array}$ \\
\hline 10 & 0.014 & 0.037 & 0.085 & 0.26 & 0.48 \\
20 & 0.030 & 0.071 & 0.16 & 0.35 & 0.66 \\
40 & 0.063 & 014 & 0.28 & 0.57 & 0.83 \\
70 & 0.11 & 0.24 & 0.43 & 0.74 & 0.92 \\
100 & 0.15 & 0.32 & 0.55 & 0.82 & 0.96 \\
150 & 0.22 & 0.44 & 0.69 & 0.89 & \\
200 & 0.30 & 0.54 & 0.75 & & \\
\hline
\end{tabular}

${ }^{7}$ NBS, Tables of Selected Values of Chemical Thermodynamic Properties, Series I, Vol. I, March, 1947 to June, 1949.

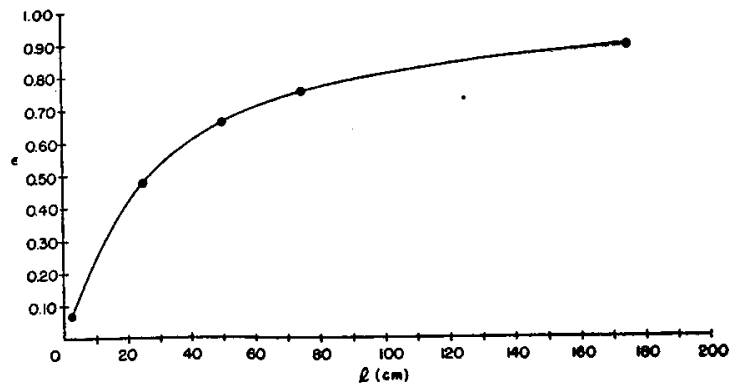

FIG. 8. Emissivity of hydrogen atoms as a function of geometrical depth ( $T=12600^{\circ} \mathrm{K}, P_{r}=20$ atmos).

necessary to consider the Lyman continuum for the temperatures used here. As seen from the figures, the continuous spectrum contributions to the emissivity increase as the temperature increases. The significant lines are located as follows: Paschen series, $\omega_{0}=5330$, 7790,9130 , 9940, and 10480 ; Balmer series, $\omega_{0}=15220$, $20580,23050,24400,25150$, and 25750 ; and Lyman series, $\omega_{0}=82200$. As may also be seen from the figures, the intensity width increases as the temperature increases, and hence discrete transition contributions also increase. In general, the continuous spectrum gives the major contribution to the emissivity at high temperatures $\left(T>9200^{\circ} \mathrm{K}\right)$, and the discrete transitions give the major contribution at low temperatures $(T$ $\approx 9200^{\circ} \mathrm{K}$ ). In a similar manner it may be shown that for a constant temperature the emissivity increases with pressure because the number density of emitters increases (fixed $l, X$ increasing) linearly with pressure; furthermore, the lines are broadened as the pressure is raised.

The data listed in Table II are obtained from such plots as are shown in Figs. 1 to 5 . These data are shown in graphical form in Figs. 6 and 7 . From these figures it is seen that the emissivity approaches unity $(\epsilon>0.95)$ for temperatures of the order of $12500^{\circ} \mathrm{K}$ and higher, with pressures of the order of 100 atmos and higher, while for temperatures of the order of $9500^{\circ} \mathrm{K}$ and lower, with pressures of the order of 10 atmos and lower, the emissivity approaches zero $(\epsilon \approx 0.05)$. The graph in Fig. 8 shows the change in the total emissivity as a function of geometrical depth for constant temperature $\left(T=12600^{\circ} \mathrm{K}\right)$ and pressure $\left(P_{T}=20\right.$ atmos). If either the temperature or pressure is decreased, the emissivity decreases and the $\epsilon$ vs $l$ curve lies under the curve shown. Similarly, if either the temperature or pressure is increased, the $\epsilon v s l$ curve lies above the curve shown.

The values of total emissivity derived by the above method can be used to estimate the magnitude of the radiant heat transfer rate for hydrogen in the temperature and optical density range considered.

The authors thank Dr. S. S. Penner for helpful discussions throughout the course of this investigation. 